\title{
Efficacy and safety of a new disposable percutaneous positioning device invented to facilitate the precision of percutaneous core needle lung biopsies: a prospective, open and randomized controlled study
}

\author{
Yunhua Xu ${ }^{1 \#}$, Jun $\mathrm{Fu}^{2 \#}$, Wenwei Cao ${ }^{3 \#}$, Li Zhu ${ }^{4}$, Yuehui Jin ${ }^{5}$, Qing Yin ${ }^{5}$, Jianding Ye ${ }^{4}$, Haiyan Zhou ${ }^{5}$ \\ ${ }^{1}$ Department of Shanghai Lung Cancer Center, Shanghai Chest Hospital, Shanghai Jiao Tong University, Shanghai, China; ${ }^{2}$ Department of \\ Radiology, Shanghai Jing'an Mental Health Center, Shanghai, China; ${ }^{3}$ Department of Science \& Education, Shanghai Jing'an Mental Health Center, \\ Shanghai, China; ${ }^{4}$ Department of Radiology, Shanghai Chest Hospital, Shanghai Jiao Tong University, Shanghai, China; ${ }^{5}$ Shanghai Chest Medical \\ Technological Co., Shanghai, China \\ Contributions: (I) Conception and design: Y Xu, J Fu, Y Jin, J Ye, H Zhou; (II) Administrative support: Y Xu, H Zhou; (III) Provision of study \\ materials or patients: Y Xu; (IV) Collection and assembly of data: Y Xu, J Fu, W Cao; (V) Data analysis and interpretation: Y Xu, J Fu, L Zhu, Q Yin; \\ (VI) Manuscript writing: All authors; (VII) Final approval of manuscript: All authors. \\ \#These authors contributed equally to this work. \\ Correspondence to: Yunhua Xu. Department of Shanghai Lung Cancer Center, Shanghai Chest Hospital, Shanghai Jiao Tong University, No. 241 \\ West Huaihai Road, Shanghai 200030, China. Email: yhxu@shchest.org; Haiyan Zhou. Shanghai Chest Medical Technological Co., No 757 Xiaonan \\ Road, Shanghai 201400, China. Email: haiyanchest@163.com.
}

Background: A new disposable percutaneous positioning device was developed which permits adjustment of the fixing puncture angle while performing a percutaneous core needle lung biopsy (CNLB). The aim of the study was to explore the positioning accuracy and clinical safety of the new device during CT-guided percutaneous CNLB.

Methods: A prospective, open and randomized controlled study with two parallel groups was conducted on 150 patients with pulmonary nodules between July 1, 2018 and June 30, 2019 including 101 males and 49 females who were divided (allocation ratio: 1:1) into a standard CNLB group without the percutaneous positioning device (control, $\mathrm{n}=75$ ) and a new percutaneous positioning device group combined with CNLB (experimental, $\mathrm{n}=75$ ). The efficacy was determined by the success rate of reaching the target location on the first percutaneous attempt, the number of CT scans performed and the time required for successful puncture positioning. Safety evaluations included complications related to percutaneous surgery and the new positioning device.

Results: The success rate of reaching the target location on the first percutaneous attempt in the experimental group was significantly higher than in the control group [96.0\% (72/75) vs. 42.7\% (32/75), $\mathrm{P}<0.001]$. Patients in the experimental group received 3.05 \pm 0.28 times CT scans on average for successful percutaneous positioning, which was significantly lower than for the control group $(3.95 \pm 1.03)(\mathrm{P}<0.001)$. The time required to complete the percutaneous positioning was significantly lower in the experimental group compared to the control group $(8.73 \pm 3.11$ vs. $12.79 \pm 4.55 \mathrm{~min}, \mathrm{P}<0.001)$. There was no significant difference in percutaneous-related complications between the two groups [48.0\% (36/75) vs. 61.3\% (46/75), $\mathrm{P}=0.101]$, except that the bleeding rate in the experimental group was lower than in the control group [26.7\% (20/75) vs. 46.7\% (35/75), $\mathrm{P}=0.032]$.

Conclusions: The use of the optimized percutaneous technology with the new positioning device significantly improved the accuracy and precision of percutaneous lung biopsies.

Trial Registration: ChiCTR-INR-17010322. 


\begin{abstract}
Keywords: New disposable positioning device; percutaneous core needle lung biopsy (CNLB); lung nodules; CTguided; precision medicine
\end{abstract}

Submitted Nov 12, 2020. Accepted for publication Jul 23, 2021.

doi: $10.21037 /$ jtd-20-3282

View this article at: https://dx.doi.org/10.21037/jtd-20-3282

\section{Introduction}

Precision diagnoses and treatments for patients with suspected lung cancer mostly depend on accurate histopathological subtyping and molecular profiling, after analysis of a biopsy specimen obtained by minimally invasive means (1). CT-guided percutaneous lung biopsy (CT-PLB) is a commonly used method for obtaining small specimens of peripheral pulmonary nodules and is also an important component of non-vascular intervention techniques in the lungs (2-4).

The surgeon must transfer the planned insertion angles from the virtual 3D image space to the patient and insert the needle accordingly. Depending on his/her confidence and the risk of damaging critical tissues, the surgeon may choose to insert the needle in a single stroke or, more typically, employ a stepwise procedure, with intermediary confirmation scans and angular adjustments, which makes the number of iterations highly dependent on the individual surgeon's skill. The iterative process of manual adjustments to needle placement and subsequent validation has three direct negative consequences: (I) increased tissue damage with each erroneous needle insertion; (II) increased radiation exposure of the patient and surgeon with each image scan, in the case of X-ray-based imaging; and (III) increased costs and equipment occupation due to the increased procedure time. In order to avoid these problems novel CT navigation systems have been introduced ranging from electromagnetic and augmented reality CT navigation systems to an optical tracking system and a multi-slice computed tomography three-dimensional digital navigated puncture technique (5-12).

Here we present a newly developed percutaneous disposable positioning device that can precisely adjust the insertion angle of the coaxial system (coaxial tube) and fix the angle of the insertion tract during the percutaneous intervention process to improve the accuracy of core needle lung biopsies (CNLBs).

We hypothesized that with the novel device, X-ray exposure and operation times as well as the incidence of complications would be markedly reduced.
We present the following article in accordance with the CONSORT reporting checklist (available at https://dx.doi. org/10.21037/jtd-20-3282).

\section{Methods}

\section{Patients}

This is a prospective, open and randomized controlled study with two parallel groups conducted in the Shanghai Chest Hospital. The experimental group were operated on with the new positioning percutaneous device to assist with CTguided percutaneous CNLB; the control group underwent standard CT-guided percutaneous CNLB (Figure 1). The trial was conducted in accordance with the Declaration of Helsinki (as revised in 2013). All preoperative patients received relevant laboratory tests, electrocardiograms (ECGs) and chest CT examinations, and were required to sign informed consent before being enrolled. The study was approved by the Ethics Committee of the Shanghai Chest Hospital (KS(Y)1676) and registered with the China Clinical Trial Center (www.chictr.org.cn, No. ChiCTRINR-17010322).

\section{Inclusion criteria}

(I) Patients of any gender aged 18 to 80 years, with a performance status (PS) score of 0 or 1 ;

(II) Peripheral pulmonary solid occupation and maximum diameters of lesions on the proposed percutaneous path $\geq 15 \mathrm{~mm}$;

(III) Parameters of a routine blood test, coagulation function test and other indicators (ECG, lung function) were normal before CNLB;

(IV) Patient agreed to join in the study and signed written informed consent.

\section{Exclusion criteria}

(I) Coagulation abnormalities or a tendency to bleed;

(II) Other diseases such as poor pulmonary functions, 


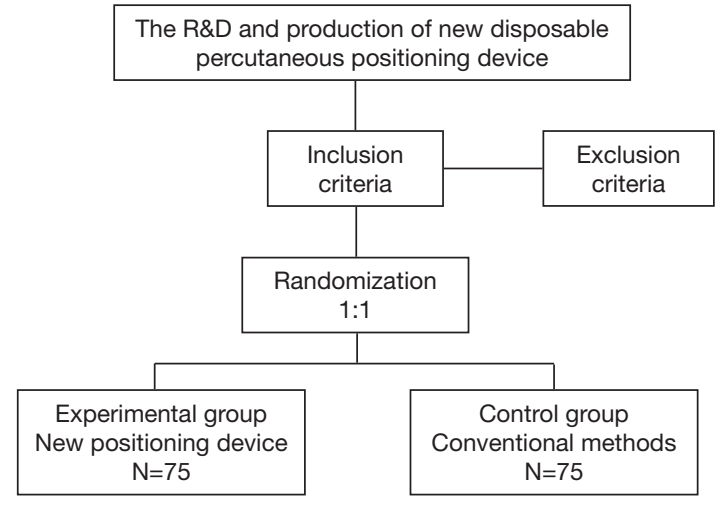

Figure 1 Flow chart of the study. R\&D, research and development.

pulmonary fibrosis, pulmonary hypertension or severe emphysema;

(III) Cardiac insufficiency, myocardial infarction or uncontrolled severe arrhythmia;

(IV) Maximum diameters of lesions on the proposed percutaneous path were $<15 \mathrm{~mm}$;

(V) Proposed percutaneous angles were parallel or vertical to the horizontal, i.e., $0^{\circ}$ or $90^{\circ}$;

(VI) Lung herpes or a pulmonary cyst on the proposed percutaneous path;

(VII) Proposed percutaneous lesions were highly suspected to be intrapulmonary vascular lesions, such as vascular malformation and aneurysms;

(VIII) Inability to tolerate the percutaneous process or uncontrolled severe cough;

(IX) Did not provide signed written informed consent.

\section{Stopping guideline}

(I) The investigator deemed it medically necessary to stop the trial;

(II) Sponsor requested to stop the trial;

(III) The ethics committee ordered the stop of the trial for some reason.

\section{Equipment}

An uCT510 16-layer spiral CT machine (United Health Imaging, UIH, China) was utilized for scans. Its specific parameters were $120 \mathrm{kV}, 150 \mathrm{~mA}$, pitch 0.9375 , rotation time $0.6 \mathrm{~s}$, thickness of layer $5 \mathrm{~mm}$ and layer spacing $5 \mathrm{~mm}$. As for biopsy, this study used core needle biopsy (CNB) that utilized the Bard $^{\circledR}$ TruGuide $^{\mathrm{TM}}$ disposable coaxial biopsy needle (specification: C1816A, Bard Peripheral, Inc., US). It is comprised of a coated needle on the outside and a coaxial core needle inside. The disposable biopsy needle (specification: MN1816, $\operatorname{Bard}^{\circledR}$ ) had two options of a tissue cutting groove length, namely 15 or $22 \mathrm{~mm}$. In the present study, we used only a needle with a $15 \mathrm{~mm}$ cutting groove. A fully automatic percutaneous biopsy gun (specification: MG1522, Bard $^{\circledR}$ Magnum $^{\mathrm{TM}}$ ) was also employed.

\section{Design of the new self-developed disposable percutaneous positioning device}

The new disposable percutaneous positioning device assists in adjusting and fixing the percutaneous angle of the biopsy needle and avoids the offset of the insertion path. It functions in conjunction with the use of coaxial biopsy needles and external protractors. The device consists of 3 integrated structures namely a positioning plate, a positioning bead and a fixed snap (Figure 2). Before injection, the positioning device is pasted onto the percutaneous point. The coaxial biopsy needle penetrates the chest through the percutaneous hole in the center of the positioning bead and the input horizontal angle is adjusted by the external protractor. Then we fastened the fixed snap around the positioning bead to lock the position of the adjusted coaxial biopsy needle.

The device had the following design characteristics: (I) the penetrating path of needles in the device could be visualized on the CT image; (II) the fixed snap that held the needle and positioning bead could strengthen the fixing function; (III) the positioning plate was made of elastic rubber material, which perfectly fitted the skin; several holes were incorporated in the plate to improve air permeability; (IV) the integrated design of the device reduced the complexity of the positioning system. It is simple and convenient to operate and can be clinically popularized compared with the IG4 electromagnetic navigation (11-14), CT 3D digital navigation or the 3D printing common-sided percutaneous template systems.

A Chinese national invention patent (No. 201811176318. $\mathrm{X}$ ) and a patent cooperation treaty (No. PCT/CN2019/1 08869) has been applied for this device. A Chinese utility model patent (No. 201821638194.8) has been granted.

\section{Conventional CT-guided percutaneous CNLB methods and the new percutaneous positioning device}

In the present study, the entire puncture process was performed by $\mathrm{Y}$. $\mathrm{Xu}$ as the first operator and assisted by 

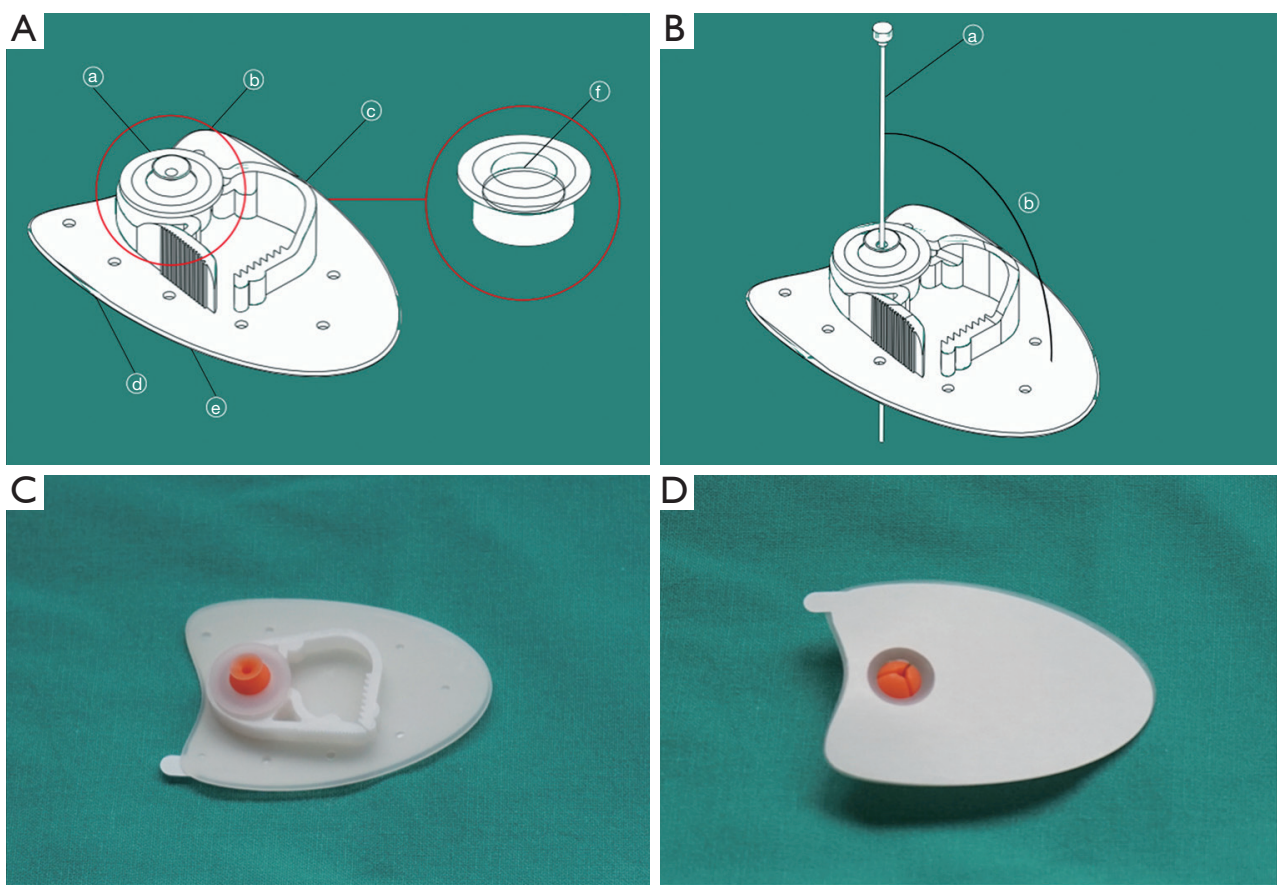

Figure 2 The new disposable percutaneous positioning device. (A) Device model before penetration (a) positioning bead, (b) pinhole, (c) fixed snap, (d) silicone plate, (e) medical adhesive plate (f) lead wire. (B) Device model after penetration (a) percutaneous needle, (b) penetrating angle. (C) Physical product, front. (D) Physical product, back.

the radiology team, including radiologists (J.D. Ye, L. Zhu), and radiology technicians (Y.B. Sun, J. Jiao and Q.M. Ni). Y. Xu is the director of the Department of Oncology and has performed more than 1,000 cases of CT-PLBs. The director, assistant directors and professionally trained technicians in the radiology team have been engaged in CT-PLB sampling for more than 10 years.

\section{Conventional CT-guided percutaneous CNLB method}

(I) The patient was placed in a flat position (supine or prone) and received their first CT scan. The proposed horizontal penetrating angle (sharp angle), the straight distance of the positioning point from the skin to pleura and other parameters were measured on the CT monitor, and then the percutaneous point was marked on the skin.

(II) After routine preparation for surgery (disinfection, towel placing), local anesthetic was injected into the pleural membrane (lidocaine).

(III) Next, following previously set parameters, the surgeon penetrated the coaxial biopsy needle through the percutaneous point of the skin into the chest until it reached the nodular lesion with the help of an external digital angle instrument, and then until the tip reached the inside of the lesion, according to previous studies (15-18).

(IV) A second CT scan was performed to determine the tip of the coaxial biopsy needle had reached the inside of the lesion and met the requirements for a subsequent cutting biopsy so that the entire percutaneous positioning process was completed. If the tip of the coaxial biopsy needle did not reach the required position, or if the tip reached the inside of the lesion but did not meet the need for a subsequent cutting biopsy, the former steps were repeated until the requirements were met.

(V) After pulling out the core coaxial biopsy needle, we kept the biopsy needle in place and inserted it from the coaxial positioning pinhole channel into the target position of the lesion. The biopsy needle was activated to cut tissue and then the biopsy needle was withdrawn with appropriate specimens attached. This operation could be repeated according to the clinical requirements for multiple sampling.

(VI) After sampling sufficient specimens, the core coaxial 

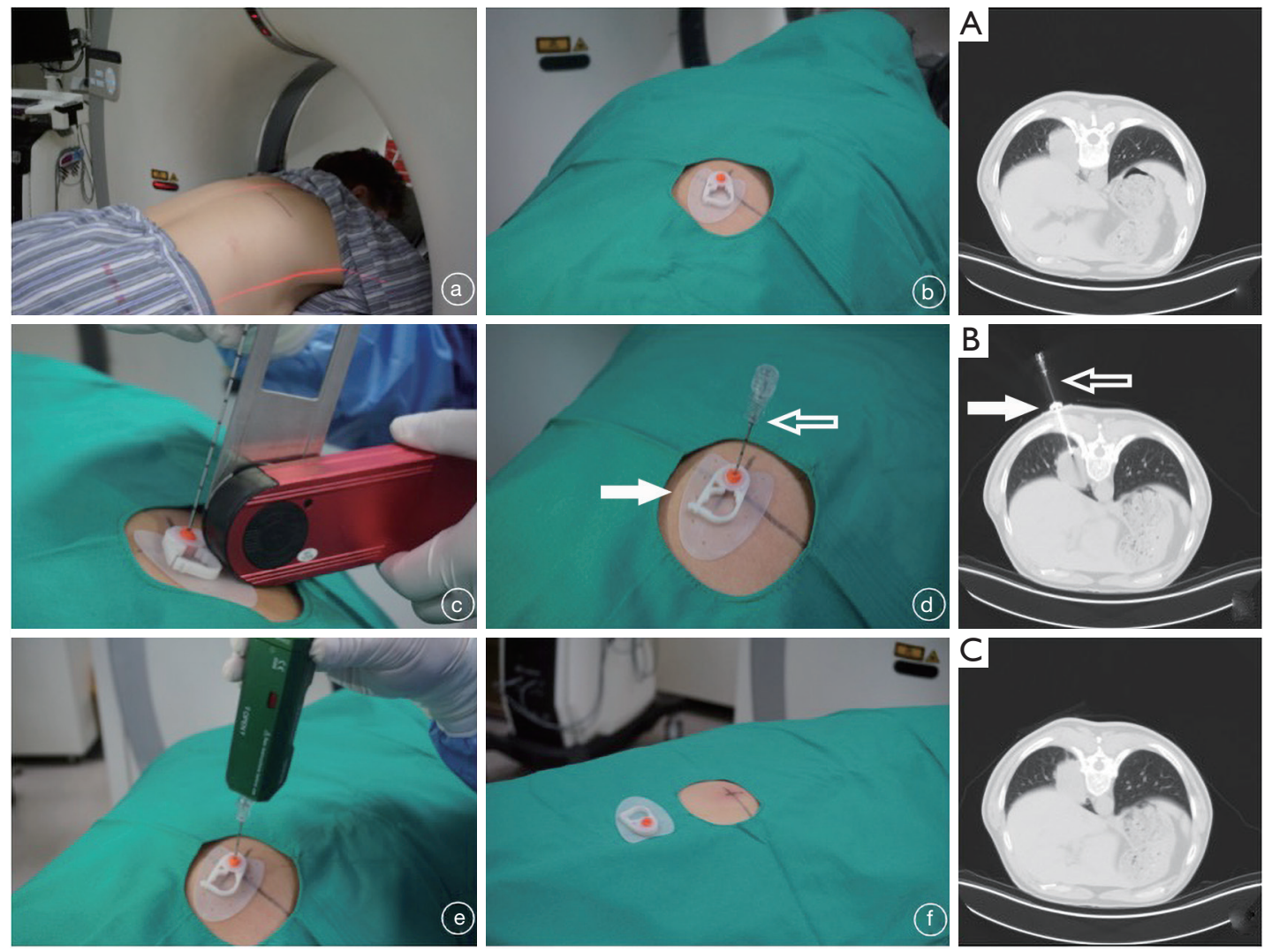

Figure 3 Instructions for the new positioning percutaneous device. Panels (a)-(f) correspond to the text description steps; ABC is the first, second and third CT scan image, respectively. (A) the status of step 1 before the operation; the patient was placed in a flat postion (prone) and received the first CT scan; (B) the status of step 4; now the coaxial biopsy needle has been placed (reaching the inside of a particular lesion), and the new device also can be seen at the same time, so that the biopsy can be performed; (C) the status of step 6 after sampling specimens; the coaxial biopsy needle was withdrawn and the postioning device was removed; finally, the patient received the last CT scan to determine that percutaneous complications had not occurred. Solid arrow, new positioning device; hollow arrow, coaxial biopsy needle.

biopsy needle was inserted inside the coat coaxial biopsy needle, spun and then the coaxial biopsy needle was withdrawn and the positioning device removed from the patient's skin.

Finally, a third CT scan was conducted to determine whether percutaneous complications had occurred (Figure 3).

\section{Instruction for the new percutaneous positioning device}

Steps (I), (II), (V) and (VI) were the same as for the conventional CNLB technique (control group). The different steps were: (III) the surgeon pasted the new positioning device to the percutaneous point on the skin, and then penetrated the coaxial biopsy needle into the chest through the percutaneous hole in the center of the positioning bead. A proper horizontal angle was obtained by adjusting the external digital angle instrument so that the needle entered the chest through the percutaneousd skin point to the targeted lesion inside. (IV) After a second CT scan, the surgeon determined whether the tip of the coaxial biopsy needle had reached the inside of a particular lesion and met the requirements for a subsequent cutting biopsy. If the procedure was deemed to be successful, fixed snaps were fastened, the coaxial biopsy needle locked and the percutaneous positioning completed. If the tip of the coaxial biopsy needle did not reach the desired position or if the tip of the needle reached the inside of the lesion but did not meet the requirements for a subsequent cutting biopsy, the steps were repeated until the requirements were met (Figure 3). 


\section{Basic demographic information and laboratory indicator measurements}

Basic demographic information was obtained from patient hospitalization medical records. All preoperative patients were subjected to laboratory tests, electrocardiogram and chest CT examinations to determine lesion sizes, locations, possible complications and other relevant information.

The primary endpoint was the success rate of reaching the target location on the first percutaneous attempt (the number of cases of reaching the predetermined location on the first percutaneous attempt/total biopsied cases) confirmed by CT scans and verified by the surgeon; the secondary endpoints were the number of CT scans performed for successful percutaneous positioning and the time required to complete percutaneous positioning. Safety assessments were divided into complications related to percutaneous surgery, which included: (I) pneumothorax; (II) bleeding; (III) pneumothorax combined with bleeding; (IV) others (including rare complications such as pleural reactions) and complications related to the new positioning device; (V) skin allergies to medical adhesives; (VI) slips due to looseness or failure to bond the device component during the biopsy process. The severity of complications was categorized into 5 degree levels: I (observation only); II (needs treatment, no prolonged hospitalization); III (treatment, extended hospitalization for at least $48 \mathrm{~h}$ ), IV (life-threatening), and V (death). Two h after biopsy and during the next day, expiratory posteroanterior chest radiographs were carried out to check for pneumothorax (19). Clinical pactice should be followed according to the severity of the recorded complications thus: (I) continue the biopsy process and subsequent observation and follow-up; (II) stop the biopsy process and conduct corresponding treatments (hemostasis treatment by drug injection or thoracic catheter drainage).

The clinical prognosis of complications was classified into 4 categories: (I) healing; (II) improvement; (III) no improvement; or (IV) unknown. All patients were followed-up by telephone consultations 1 week after their percutaneous operations.

\section{Sample size}

Considering previous studies, the success rate of puncture by a one-time attempt was $50 \%$ in the control group $(20,21)$ and the superior surgeon in our team had a $60 \%$ success rate in clinical practice. We assumed, that the success rate of puncture by a one-time attempt would be $60 \%$ in the control and $95 \%$ in the experimental group. Group sample sizes of 62 in each group achieved a $90.19 \%$ power to detect a difference between the group proportions of 0.35 . The control group proportion was 0.60 and the experimental group proportion was assumed to be 0.75 under the null hypothesis and 0.95 under the alternative hypothesis. The test statistic used was a one-sided $\mathrm{Z}$ test (unpooled). The significance level of the test was set at 0.05 . In the present study, 72 cases were recruited into each of the 2 groups to allow for an expected $15 \%$ drop-out rate.

\section{Randomization}

The study participants were divided into an experimental group (use of the new positioning device) and a control group without the positioning device by simple randomization with a 1:1 allocation ratio. The authors assigned Yan Pan, who was not involved in the intervention of this study, to be in charge of patient allocation. The sequence was generated using a random number table. The sealed opaque envelope was used to conceal the sequence until interventions were assigned.

\section{Statistical methods}

The basic characteristics of the baseline (before percutaneous biopsy) and biopsy efficacy and safety after percutaneous biopsy were compared between the experimental and control groups. Continuous normally distributed variables were compared using a $t$-test (or K-S test), and categorized variables such as gender and pathological diagnosis were compared using a chi-squared test. Since there was no dropout of patients in the study, all statistical evaluations were conducted on the per protocol (PP) population. The data were statistically analyzed using SPSS ver. 20.0 software and a $\mathrm{P}$ value $<0.05$ was considered to be a statistically significant finding.

\section{Results}

\section{The condition of lesions and percutaneous pathology results}

A total of 168 patients with pulmonary nodules were recruited in the Oncology Department of Shanghai Chest Hospital between July 1, 2018 and June 30, 2019. Finally, 150 patients who met the criteria were included in the study, including 101 males and 49 females, who were randomly 
Table 1 Patient characteristics at baseline and procedure-related characteristics

\begin{tabular}{lccc}
\hline & Experimental group & Control group & $\mathrm{t}\left(\chi^{2}\right)$ value \\
\hline Age (years) & $65 \pm 8$ & $63 \pm 11$ & $\mathrm{t}=1.014$ \\
Gender & & & \\
Man & 56 & 45 & 30.312 \\
Woman & 19 & 30 & $\chi^{2}=3.667$ \\
Diagnosis of disease & & 52 & 0.055 \\
Unknown & 54 & 23 & $\chi^{2}=0.129$ \\
Defined & 21 & 0.720 \\
\hline
\end{tabular}

The diagnosis in the table refers to clear lung cancer pathology diagnosis before the operation but some patients required another biopsy due to their current condition or treatment requirements.

divided into 2 groups, with 75 patients in each group (Figure 1). There was no statistical difference in age and disease diagnosis between the 2 groups (Table 1).

The majority of lesions in patients receiving the new positioning device were located in the upper left lung (22, $29 \%)$, followed by the lower right lung $(20,27 \%)$ or the upper right lung $(15,20 \%)$. The lesions in the control group were concentrated in the lower right lung $(19,25 \%)$, followed by the upper right lung $(18,24 \%)$ and the upper left lung (16, 21\%) (Figure 4A). Except for the maximum diameter value of the lesions in the percutaneous path, the straight distance of the positioning point from the skin to the pleura and from the pleura to the outer edge of the lesion, and the total length of the coaxial biopsy needle in the chest and the horizontal angle of the coaxial biopsy needle appeared to be greater than in the control group, but the differences were not statistically significant. In addition, the percutaneous pathology results were compared and the results of pathological tissues acquired from the 2 patient groups were different. Squamous cell carcinoma and lung or necrotic tissue accounted for a larger proportion in the experimental group than in the control group, but the proportion of small-cell carcinomas and pulmonary tuberculosis tissues was much lower in the experimental group than that in the control group (Figure 4B).

\section{The effectiveness of the new percutaneous device compared to the control routine procedure}

We compared the efficacy, including the primary and secondary endpoints, between the 2 groups and found the success rate of reaching the target location on the first percutaneous attempt in the experimental group was $96.0 \%$ higher than in the control group (42.7\%), and the actual number of puncture operations was less $(1.05 \pm 0.28)$ in the experimental group than in the control group $(1.95 \pm 1.03)$.

The secondary endpoints were the number of CT scans performed for successful puncture positioning of the coaxial biopsy needle and the whole operation took less time in the experimental group than in the control group (both $\mathrm{P}<0.05$ ). The times required to finish the entire percutaneous positioning procedure and the total operation times were shorter in the experiment group than in the control group (both $\mathrm{P}<0.05$; Table 2).

Thus, these endpoints reached the goal of our study, because the shorter operation times and fewer actual percutaneous times were beneficial for patients, particularly in reducing the pain and discomfort induced by the operation. In addition, the reduced number of CT scans resulted in a reduced radiation exposure for each patient and the surgeon.

\section{Comparison of complications in the two groups}

In terms of safety indicator I (complications related to percutaneous surgery), the results suggested that the incidence of complications in the experimental group was lower than in the control group, but the findings did not reach statistical significance $[48.0 \%(36 / 75)$ vs. $61.3 \%(46 / 75), \mathrm{P}=0.101]$. The incidence of bleeding complications in the 2 groups (including bleeding only and bleeding combined with pneumothorax) was $26.7 \%$ (20/75) vs. 46.7\% (35/75), respectively and significantly different $(\mathrm{P}=0.032)$. The incidence of pneumothorax complications (pneumothorax only and pneumothorax combined with bleeding) was $30.7 \%(23 / 75)$ and $21.3 \%$ 

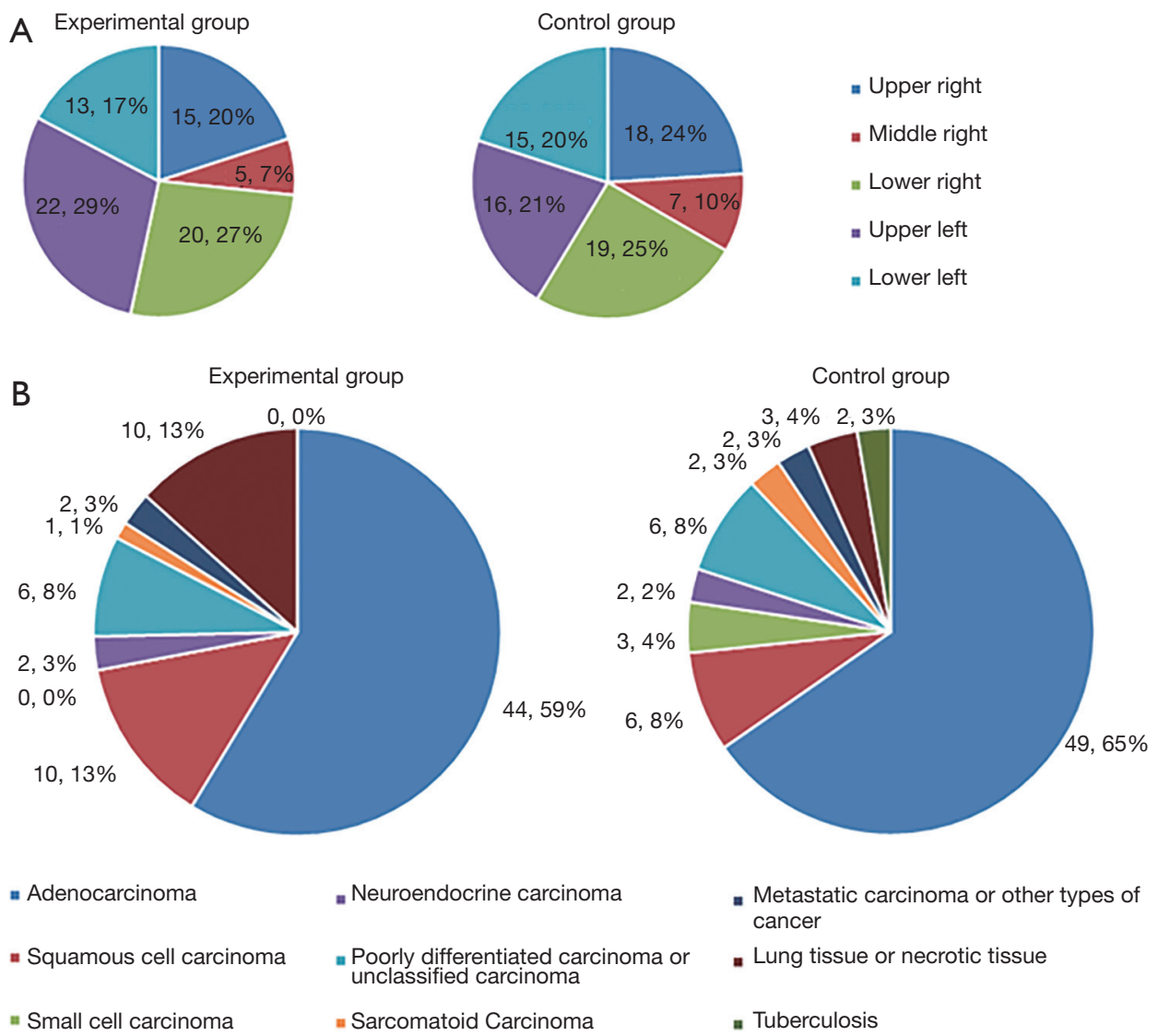

„Neuroendocrine carcinoma

" Poorly differentiated carcinoma or unclassified carcinoma
- Metastatic carcinoma or other types of cancer
- Lung tissue or necrotic tissue
n Tuberculosis

« Sarcomatoid Carcinoma

Figure 4 The results of location of lesions and percutaneous pathology were compared between the two groups. (A) Comparison of the location of lesions between the 2 groups; (B) comparison of percutaneous pathology between the 2 groups.

Table 2 Device efficacy and related indictors

\begin{tabular}{|c|c|c|c|c|}
\hline & Experimental group & Control group & $\mathrm{t}\left(\chi^{2}\right)$ value & $P$ value \\
\hline $\begin{array}{l}\text { The number of CT scans performed for successful coaxial biopsy needle } \\
\text { puncture positioning }\end{array}$ & $2.05 \pm 0.28$ & $2.95 \pm 1.03$ & -6.989 & $<0.001$ \\
\hline The time of finishing the entire percutaneous positioning procedure (min) & $8.73 \pm 3.11$ & $12.79 \pm 4.55$ & -5.555 & $<0.001$ \\
\hline Total whole surgery time (min) & $13.73 \pm 3.94$ & $17.68 \pm 5.17$ & -4.188 & $<0.001$ \\
\hline
\end{tabular}

$(16 / 75)$, respectively $(\mathrm{P}=0.193)$ but there was no significant difference in the severity of complications between the 2 groups $(\mathrm{P}=0.559)$. In both groups, 2 patients reported IIIdegree severity complications, and both occurred after the completion of CNLB. After appropriate treatment the patients condition greatly improved. All patients did not experience any new complications during the first week after their biopsies and the original complications disappeared. No case presented with symptoms of safety indicator II (complications related to new positioning devices) in the 
Table 3 Percutaneous lung biopsy for all adverse events

\begin{tabular}{|c|c|c|c|c|c|c|c|c|}
\hline & \multicolumn{3}{|c|}{ Complications category (a) } & \multicolumn{5}{|c|}{ Severity of complications (b) } \\
\hline Control group (n, \%) & $11(14.7)$ & 30 (40.0) & $5(6.7)$ & 26 (56.5) & $18(39.1)$ & $2(4.4)$ & 0 & 0 \\
\hline
\end{tabular}

(a), Complication category: $\chi^{2}=6.863, \mathrm{P}=0.032$; the difference was statistically significant. (b), The severity of complications: $\chi^{2}=0.994$, $\mathrm{P}=0.608$; the difference was not statistically different.

experimental group (Table 3).

\section{Discussion}

At present, CNLB is a minimally invasive operation widely used for the pathological diagnosis of lung disease and $40-60 \%$ of patients would be expected to reach a predetermined position with one percutaneous attempt using the traditional method $(22,23)$.

The effectiveness indicators, including the rate of reaching a target location on the first percutaneous approach, the number of CT scans performed for successful percutaneous positioning, and the time required to complete the percutaneous positioning in this study showed that outcomes in the experimental group were significantly better than in the control group (Table 2). The application of the new positioning device not only reduced the possibility of repeated percutaneous invasions and shortened the operation time but also reduced the occurrence of bleeding complications.

In addition, we evaluated the safety of the new positioning device for the skin. The experimental results showed that none of the patients in the experimental group reported negative conditions, proving the reliability of the new device. In the experimental group, we also encountered the following cases, which are not uncommon in clinical practice and are extremely difficult to solve (24). These cases included: (I) small diameters of lesions on the percutaneous path $(15-20 \mathrm{~mm})$; (II) the drill percutaneous angle $\left(<30^{\circ}\right.$ penetrating angle); (III) irregular distribution of the apparent necrosis-like substance in the center of a lesion; and (IV) vascular distribution inside the lesion in the percutaneous path (Figure 5). The operators also demonstrated the effectiveness of the new positioning device by successfully conducting a biopsy under these adverse conditions.

CNLB-related complications mainly focus on pneumothorax, bleeding and/or a combination of both (25-29). The results showed that the incidence of complications in the experimental group was apparently lower than in the control group, though the findings did not reach statistical significance. However, a comparison of bleeding complications revealed significant differences.

It should be pointed out that the present study had a number of limitations. Differences in the size and location of lesions can cause difficulties during percutaneous surgery and although patients were randomized further analysis will be required on whether the effectiveness and safety of percutaneous biopsies assisted by the novel positioning device might change according to different lesion characteristics.

\section{Conclusions}

The clinical application of the new positioning device improved the accuracy of CT-PLB, shortened operation times and reduced the occurrence of bleeding complications. 

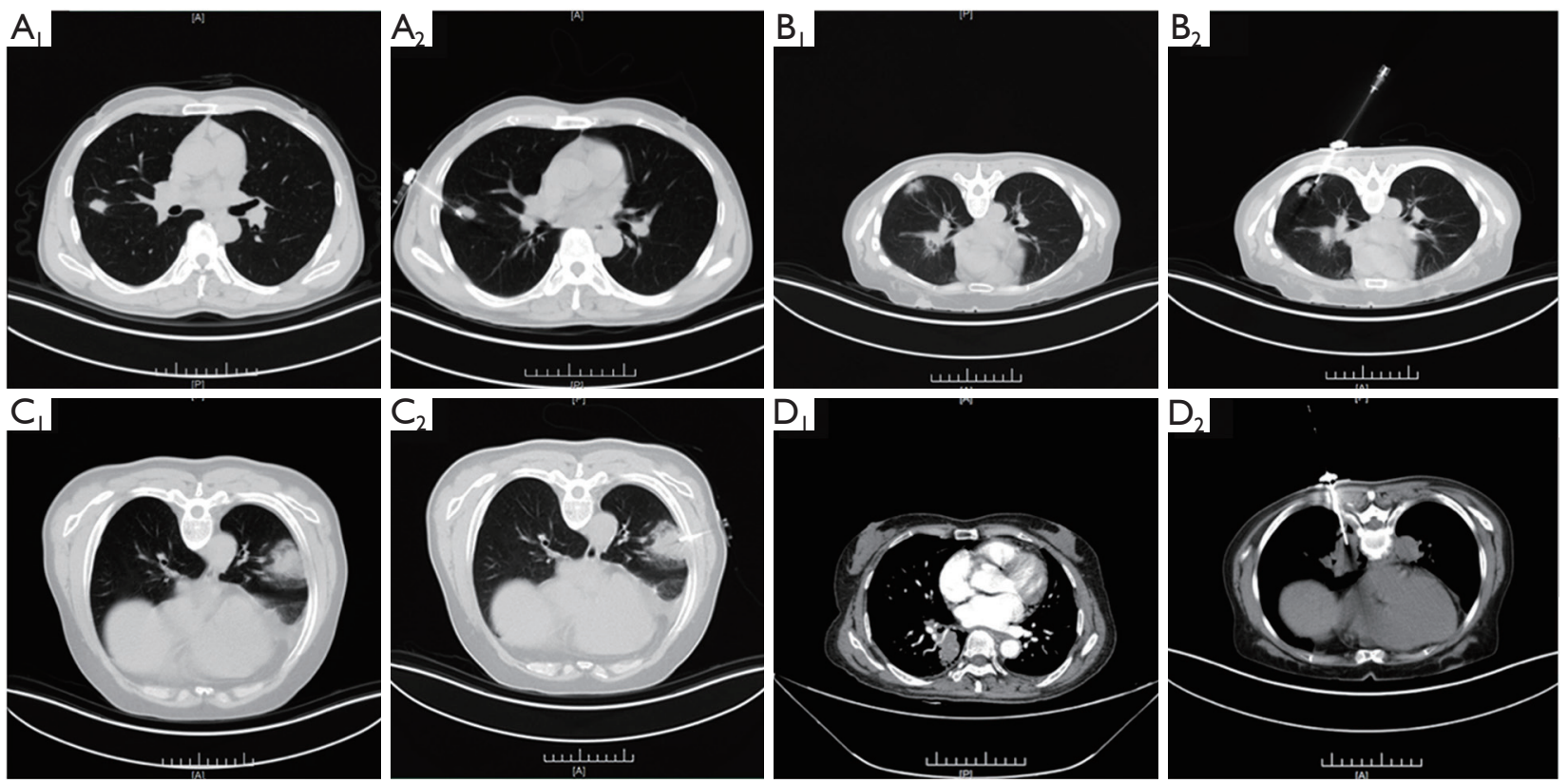

Figure 5 CT images of the new disposable percutaneous positioning device used in uncommon and difficult clinical cases. (A) small diameter of the lesions on the percutaneous path $(15-20 \mathrm{~mm})$ (CT scan); (B) the drill percutaneous angle ( $<30^{\circ}$ penetrating angle to ground) (CT scan); (C) irregular distribution of the apparent necrosis-like substance in the center of a lesion (CT scan); (D) vascular distribution inside the lesion in the percutaneous path (contrast-enhanced CT). A1,B1,C1,D1: The first CT scan image shows the location of the lesion. A2,B2,C2,D2: puncture positioning was completed and the coaxial biopsy needle reached the lesion as revealed by the CT image, then a cut biopsy was performed.

\section{Acknowledgments}

We thank the following investigators for their grateful help: Mingzhou Zhou (Shanghai Chest Medical Technological Co.), Yan Pan (Department of Pharmacy, Shanghai Chest Hospital, Shanghai Jiao Tong University), Yanbing Sun, Jing Jiao and Qiming Ni (Department of Radiology, Shanghai Chest Hospital, Shanghai Jiao Tong University), Shun Lu, Shengping Shen and Yaxian Yao (Department of Shanghai Lung Cancer Center, Shanghai Chest Hospital, Shanghai Jiao Tong University), Haijin Liu (Department of Nursing, Shanghai Chest Hospital, Shanghai Jiao Tong University).

Funding: This study was supported by the foundation of Science and Technology Commission of Shanghai Municipality Project (grant no. 19140902600), Shanghai Chest Hospital Project (grant no. 2019YNJCM06), Clinical Research Plan of SHDC (grant no. 16CR3102B), Shanghai Chest Hospital Project of Collaborative Innovation Grant (grant nos. YJXT20190210 and YJXT20190210Z) and Shanghai Municipal Health Commission Project (grant no. 201940221). The funders had no role in the study design, data collection or analysis, decision to publish or preparation of the manuscript.

\section{Footnote}

Reporting Checklist: The authors have completed the CONSORT reporting checklist. Available at https://dx.doi. org/10.21037/jtd-20-3282

Trial Protocol: Available at https://dx.doi.org/10.21037/jtd20-3282

Data Sharing Statement: Available at https://dx.doi. org/10.21037/jtd-20-3282

Conflicts of Interest: All authors have completed the ICMJE uniform disclosure form (available at https:// dx.doi.org/10.21037/jtd-20-3282). YHJ, QY and HYZ are members of the staff of the Shanghai Chest Medical Technological Company. YHX, YHJ, QY and HYZ have patents relevant to this manuscript. The other authors have 
no conflicts of interest to declare.

Ethical Statement: The authors are accountable for all aspects of the work in ensuring that questions related to the accuracy or integrity of any part of the work have been appropriately investigated and resolved. The trial was conducted in accordance with the Declaration of Helsinki (as revised in 2013). All preoperative patients received relevant laboratory tests, electrocardiograms and chest CT examinations, and were required to sign informed consent before being enrolled. The study was approved by the Ethics Committee of our Hospital (kS (Y) 1676) and registered with the China Clinical Trial Center (www.chictr. org.cn, No. ChiCTR-INR-17010322).

Open Access Statement: This is an Open Access article distributed in accordance with the Creative Commons Attribution-NonCommercial-NoDerivs 4.0 International License (CC BY-NC-ND 4.0), which permits the noncommercial replication and distribution of the article with the strict proviso that no changes or edits are made and the original work is properly cited (including links to both the formal publication through the relevant DOI and the license). See: https://creativecommons.org/licenses/by-nc-nd/4.0/.

\section{References}

1. Chinese Thoracic Society; Chinese Alliance against Lung Cancer. Chinese expert consensus statement on issues related to small specimen sampling of lung cancer. Endosc Ultrasound 2017;6:219-30.

2. Birchard KR. Transthoracic needle biopsy. Semin Intervent Radiol 2011;28:87-97.

3. Faiella E, Frauenfelder G, Santucci D, et al. Percutaneous low-dose CT-guided lung biopsy with an augmented reality navigation system: validation of the technique on 496 suspected lesions. Clin Imaging 2018;49:101-5.

4. Tsai IC, Tsai WL, Chen MC, et al. CT-guided core biopsy of lung lesions: a primer. AJR Am J Roentgenol 2009;193:1228-35.

5. Lal H, Neyaz Z, Nath A, et al. CT-guided percutaneous biopsy of intrathoracic lesions. Korean J Radiol 2012;13:210-26.

6. Arnolli MM, Hanumara NC, Franken M, et al. An overview of systems for CT- and MRI-guided percutaneous needle placement in the thorax and abdomen. Int $\mathrm{J}$ Med Robot 2015;11:458-75.

7. Grand DJ, Atalay MA, Cronan JJ, et al. CT-guided percutaneous lung biopsy: comparison of conventional CT fluoroscopy to CT fluoroscopy with electromagnetic navigation system in 60 consecutive patients. Eur J Radiol 2011;79:e133-6.

8. Grasso RF, Faiella E, Luppi G, et al. Percutaneous lung biopsy: comparison between an augmented reality CT navigation system and standard CT-guided technique. Int J Comput Assist Radiol Surg 2013;8:837-48.

9. Moncharmont L, Moreau-Gaudry A, Medici M, et al. Phantom evaluation of a navigation system for outof-plane CT-guided puncture. Diagn Interv Imaging 2015;96:531-6.

10. Schubert T, Jacob AL, Pansini M, et al. CT-guided interventions using a free-hand, optical tracking system: initial clinical experience. Cardiovasc Intervent Radiol 2013;36:1055-62.

11. Hao W, Chen Y, Shao G. Clinical application of IG4 electromagnetic navigation system-assisted CT-guided percutaneous needle biopsy of pulmonary nodules. Journal of Interventional Radiology 2016;25:682-5.

12. Zhang K. The application of MSCT three-dimensional digital navigated puncture technique in percutaneous puncture lung biopsy. Journal of Interventional Radiology 2016;25:779-83.

13. Wang G, Chen B, Zhang Y. Clinical application of 3D printing coplanar puncture template assisted CT guided percutaneous biopsy of primary lung small nodules. Cancer Progression 2017;15:1003-7.

14. Tao G, Sun Y. Application Value Study of Four-dimensional CT in Percutaneus Aspiration Lung Biopsy. Chinese Journal of CT and MRI 2016;14:50-2.

15. Greif J, Marmor S, Schwarz Y, et al. Percutaneous core needle biopsy vs. fine needle aspiration in diagnosing benign lung lesions. Acta Cytol 1999;43:756-60.

16. Laurent F, Latrabe V, Vergier B, et al. CT-guided transthoracic needle biopsy of pulmonary nodules smaller than $20 \mathrm{~mm}$ : results with an automated 20-gauge coaxial cutting needle. Clin Radiol 2000;55:281-7.

17. Westcott JL, Rao N, Colley DP. Transthoracic needle biopsy of small pulmonary nodules. Radiology 1997;202:97-103.

18. Ohno Y, Hatabu H, Takenaka D, et al. CT-guided transthoracic needle aspiration biopsy of small $(<$ or $=20$ $\mathrm{mm}$ ) solitary pulmonary nodules. AJR Am J Roentgenol 2003;180:1665-9.

19. Kinoshita F, Kato T, Sugiura K, et al. CT-guided transthoracic needle biopsy using a puncture sitedown positioning technique. AJR Am J Roentgenol 
2006;187:926-32.

20. Li J, Li Y, Wang X. The Clinical Application of Puncture Locating Device of Three-dimensional Concept of Whole Body Guided by CT. Medical Innovation of China 2014;11:4-7.

21. Li J, Wang X, Lei M, et al. The Research and development of puncture locating device of three-dimensional concept of whole body guided by CT and the research of lung puncture in clinical application. China Medical Equipment 2015;12:29-33

22. Chellathurai A, Kanhirat S, Chokkappan K, et al. Technical note: CT-guided biopsy of lung masses using an automated guiding apparatus. Indian J Radiol Imaging 2009;19:206-7.

23. Laspas F, Roussakis A, Efthimiadou R, et al. Percutaneous CT-guided fine-needle aspiration of pulmonary lesions: Results and complications in 409 patients. J Med Imaging Radiat Oncol 2008;52:458-62.

24. Khan MF, Straub R, Moghaddam SR, et al. Variables affecting the risk of pneumothorax and intrapulmonal

Cite this article as: $\mathrm{Xu} \mathrm{Y,} \mathrm{Fu} \mathrm{J,} \mathrm{Cao} \mathrm{W,} \mathrm{Zhu} \mathrm{L,} \mathrm{Jin} \mathrm{Y,} \mathrm{Yin} \mathrm{Q,} \mathrm{Ye}$ J, Zhou H. Efficacy and safety of a new disposable percutaneous positioning device invented to facilitate the precision of percutaneous core needle lung biopsies: a prospective, open and randomized controlled study. J Thorac Dis 2021;13(8):4965-4976. doi: 10.21037/jtd-20-3282 hemorrhage in CT-guided transthoracic biopsy. Eur Radiol 2008;18:1356-63.

25. Boskovic T, Stanic J, Pena-Karan S, et al. Pneumothorax after transthoracic needle biopsy of lung lesions under CT guidance. J Thorac Dis 2014;6 Suppl 1:S99-S107.

26. Winokur RS, Pua BB, Sullivan BW, et al. Percutaneous lung biopsy: technique, efficacy, and complications. Semin Intervent Radiol 2013;30:121-7.

27. Aktaş AR, Gözlek E, Yılmaz Ö, et al. CT-guided transthoracic biopsy: histopathologic results and complication rates. Diagn Interv Radiol 2015;21:67-70.

28. Song YS, Park CM, Park KW, et al. Does antiplatelet therapy increase the risk of hemoptysis during percutaneous transthoracic needle biopsy of a pulmonary lesion? AJR Am J Roentgenol 2013;200:1014-9.

29. Tachibana K, Nakazato Y, Tsuchida S, et al. Immediate cytology improves accuracy and decreases complication rate in real-time computed tomography-guided needle lung biopsy. Diagn Cytopathol 2013;41:1063-8. 